Physiological behaviour of cells

Analytical Physiology of Cells and Developing Organisms. By B. C. Goodwin. Pp. 249. (Academic: London and New York, 1977.) £8.50: \$18.50.

THE aim of this book is to present a picture of the physiological behaviour of cells in terms of molecular biology and mathematical models. As the author acknowledges, the choice of material is not comprehensive and reflects his own interests and prejudices. In the first three chapters, Goodwin examines aspects of the behaviour of individual cells. The metabolic network, the adaptation time of which is of the order of seconds, is considered in terms of allosteric enzymes and endproduct inhibition. This network is contrasted with the epigenetic one with longer adaptation times (minutes to hours) and in which gene activation and protein synthesis are now major elements. The occurrence of feedback loops in both kinds of systems can lead to complex dynamics such as oscillations. Analogies with statistical thermodynamics are drawn; and this rather formal analysis is extended to the cell cycle.
In considering the various internal networks involved, the important point is made that there may be no single reaction controlling the entry of a cell in to the cell cycle: emphasis should be placed instead on a mixture of probabilistic and deterministic processes. The treatment of the cell cycle is, however, unsatisfactory since it provides an inadequate review of current ideas and experiments. For example, although mechanisms based on chalones which are inhibitory are considered, there is little reference to molecules exerting a positive control, for which there is increasing evidence. A nice chapter on biological clocks follows, with an explanation of Winfree's ingenious approach to the eclosion clocks in Drosophila. Here, mathematical analysis leads to experiments that could not really be conceived of without that analysis. Unfortunately, most of the other analyses in the book do not seem to have a similar experimental spin-off.

Pattern formation and its relation to gradients and positional information is given quite detailed attention. Emphasis is placed on periodic processes and a membrane wave model is put forward. A formal model to account for the properties of the polar coordinate model of French, Bryant and Bryant is proposed. Goodwin believes in the next few years we will see the emergence of new dynamical models in which waves and periodicities will feature very strongly. He recognises that gradienttype models of positional information shift the major problem of pattern formation into the area of how the gradients are interpreted by the cells in terms of gene activity, but gives this central theoretical problem inadequate attention.

In the final chapter, he treats the organism as a cognitive and cooperative system. He treats the cell as if it had knowledge about the world which is contained in its control circuits. He also wishes to regard sharp transitions of state at different levels of organisation as being basically similar and analogous to phase transitions.

Goodwin is always worth reading for his independence and insights, but overall this book is disappointing. The mathematics seems to add rather little and too seldom leads to experiments. The approach is not didactic or comprehensive. For theoreicians, however, it is essential.

Lewis Wolpert

Lewis Wolpert is Professor of Biology as Applied to Medicine at the Middlesex Hospital Medical School, London, UK.

\section{Quantum theory}

Qualitative Methods in Quantum Theory. By A. B. Migdal. Pp. 435. (W. A. Benjamin: Reading, Massachusetts and London, 1977.) \$21.50.

"THE solution of most problems in theoretical physics begins with the application of the qualitative methods which constitute the most attractive and beautiful characteristic of this discipline. By 'qualitative methods' we mean dimensional estimates and estimates made by using simple models, the investigation of limiting cases where one can exploit the smallness of some parameter, the use of the analytic properties of physical quantities, and finally the derivation of consequences from the symmetry properties, that is, the invariance relative to various transformations (eg Lorentz or isotopic invariance). However, as experience in the classroom shows, it is just these aspects of theoretical physics which are most difficult for the beginner." So Academician Migdal introduces the new edition of his book, the first three chapters of which were published in much the same form as in Approximation Methods in Quantum Mechanics (Izdatelstvo Nauka, 1966; English translation, W. A. Benjamin, 1969).

Migdal is a leading exponent of the Landau school of theoretical physics, the members of which have been brought up to know all nine volumes of Landau and Lifshitz. So in this book we have examples from a wide range of subjects. The first chapter covers mainly models in atomic physics. Chapter two is on perturbation theory, and chapter three is on the WKB method and the quasi-classical approximation, a subject which is not normally given much space in textbooks. Chapter four is on analyticity and dispersion relations. Chapter five is on quasi-particle methods in the manybody problem, with applications to quantum fluids and nuclear matter. Chapter six gives a qualitative physical treatment of renormalisation, and a discussion of the Gell-Mann-Low equation and asymptotic freedom.

This book should be required reading for any serious graduate student in theoretical physics.

Norman Dombey

Norman Dombey is Reader in Theoretical Physics at the University of Sussex, UK.
BOOKS

ON PURE

AND APPLIED SCIENCE

Books reviewed or mentioned in this journal are available from stock.

Catalogues on application. Please state interests.

\section{SCIENTIFIC LIBRARY}

ANNUAL SUBSCRIPTION from $\$ 7.00$

Reduced rates for multiple subscriptions

Available in U.K. only

Prospectus free on request

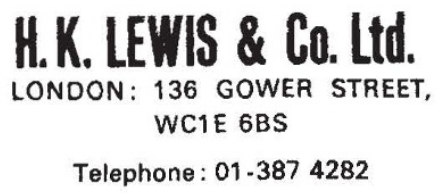

Telephone: 01 -387 4282 